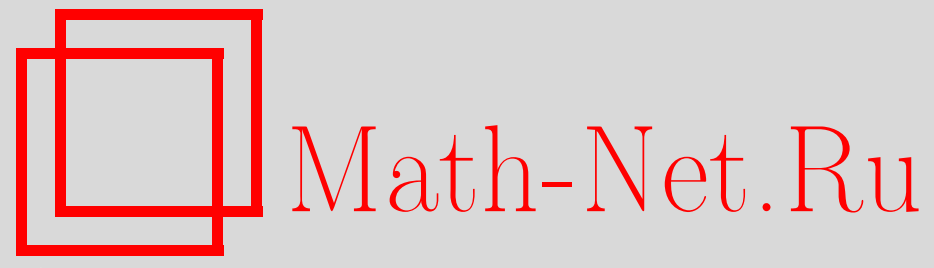

E. M. Artemova, E. V. Vetchanin, Control of the motion of a circular cylinder in an ideal fluid using a source, Vestn. Udmurtsk. Univ. Mat. Mekh. Komp. Nauki, 2020, Volume 30, Issue 4, 604-617

DOI: https://doi.org/10.35634/vm200405

Use of the all-Russian mathematical portal Math-Net.Ru implies that you have read and agreed to these terms of use

http: //www. mathnet.ru/eng/agreement

Download details:

IP : 3.95 .254 .165

April 26, 2023, $11: 16: 46$ 
MSC2010: 76Bxx, 70Exx, 34H15

\author{
(C) E. M. Artemova, E. V. Vetchanin
}

\title{
CONTROL OF THE MOTION OF A CIRCULAR CYLINDER IN AN IDEAL FLUID USING A SOURCE
}

The motion of a circular cylinder in an ideal fluid in the field of a fixed source is considered. It is shown that, when the source has constant strength, the system possesses a momentum integral and an energy integral. Conditions are found under which the equations of motion reduced to the level set of the momentum integral admit an unstable fixed point. This fixed point corresponds to circular motion of the cylinder about the source. A feedback is constructed which ensures stabilization of the above-mentioned fixed point by changing the strength of the source.

Keywords: control, ideal fluid, feedback, motion in the presence of a source.

DOI: $10.35634 / \mathrm{vm} 200405$

\section{Introduction}

The problem of controlling the motion of a rigid body in a fluid is a classical hydrodynamics problem and has been actively discussed recently in relation to problems of mobile robotics. Screw propellers $[31,54]$ and water jet thrusters $[41,45]$ are conventional and well-studied devices used for propulsion in a fluid. Other propulsion methods considered in the literature include motion control by means of internal mechanisms [7, 8, 17,42,52,59] and by deforming the body $[26,28,40]$. A detailed review of the literature devoted to the analysis of the dynamics of aquatic mobile robots is presented in [60].

The simplest model which makes it possible to obtain qualitative conclusions on the inertial and controlled dynamics of a rigid body is the model of an ideal fluid. Based on this model, the authors of $[36,37]$ showed the possibility of self-propulsion of a hydrodynamically asymmetric body in a fluid by displacing the internal moving mass along a self-intersecting contour. Further development of the ideas of [36,37] was carried out in [30] and experimental evidence of them was provided in [33]. This model was also used to study the control of body motion by rotating internal rotors $[15,61]$, moving the internal mass and rotating the rotor in the presence of constant circulation [59], by rotating the gyrostat and changing circulation using the Flettner rotor $[29,53]$.

In addition to control by changing circulation, we also consider control using sources and sinks. We recall that a source/sink is a singular point of the fluid in the punctured neighborhood of which the flow remains potential and the streamlines are radially divergent straight lines [34]. The model of a point source was applied in calculating the characteristics of airfoils with systems for the control of the boundary layer $[21,48,57]$. In particular, the problem of stabilizing the position of a vortex arising above the Kasper wing [27] was addressed in [48]. In mathematical models constructed in $[21,48,57]$, the point source was located on the wing surface.

We note that the analysis of the motion of point singularities (sources, vortices, vortex sources, dipoles) in a fluid and of their interaction with rigid bodies is interesting in its own right. In particular, the motion of $N$ sources was examined in $[5,18]$. Special attention was also given to models describing the motion of point vortices on a plane $[6,11,39]$, in general domains of a plane [19], on a sphere [10], curvilinear surfaces [9,35] and under the action of external periodic perturbation $[20,62,63]$. More complex models describing the motion of vortex sources and 
dipoles were treated in $[3,4,13,49,58]$. An analysis of the joint motion of point vortices and a smooth rigid body was made in $[14,51,56]$. In $[28,43,46]$, based on the Kutta-Chaplygin condition $[16,38]$, the separation of point vortices from the sharp edges of a rigid body was modeled.

In this paper, we consider the problem of controlling the motion of a circular foil in an ideal fluid by changing the strength of the point source. In contrast to [21,48,57], the source is located at some distance from the boundary of the foil and is not connected to it. In Section 1 we derive equations of motion of a balanced circular foil in the field of a source whose strength and position are given functions of time. In Section 2 we show that, in the case of a fixed source of constant strength, the system admits a Lyapunov unstable solution corresponding to motion of the geometric center of the foil in a circle at whose center the source is located. It turns out that this motion of the foil can be stabilized by changing the strength of the source.

We implement the control of the strength of the source by means of feedback. For synthesis of feedback, we use an approach based on the linearization of equations of motion which was applied, in particular, in the problems of stabilizing an inverted pendulum as applied to stabilization of a gyroscooter (Segway) $[2,44]$. In this paper, feedback is given in the form of a linear function of phase variables. Such a form of control is applied in $[12,23,24,32]$. We note that feedback can be nonlinear as well [22].

\section{$\S 1$. Equations of motion}

Consider the plane-parallel motion of a balanced circular foil of radius $R$ in an ideal incompressible fluid in the presence of a source of strength $q$. For this system we make the following assumptions:

$1^{\circ}$ The motion of the fluid is potential.

$2^{\circ}$ The circulation around any closed contour enclosing the foil is zero.

$3^{\circ}$ The motion of the source is given by some function of time.

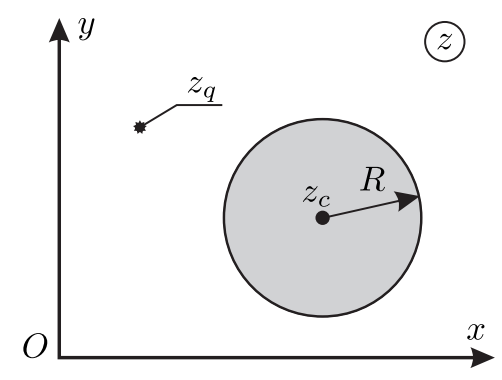

Figure 1: A circular foil and a source in a fluid

To describe the motion of the system, we introduce a fixed (inertial) coordinate system $O x y$ in which the fluid is at rest at infinity (see Fig. 1). With the coordinates $(x, y)$ we associate a complex variable $z=x+i y$. We specify the position of the center of mass of the foil as $z_{c}=x_{c}+i y_{c}$ and the position of the source as $z_{q}=x_{q}+i y_{q}$.

By virtue of assumption $1^{\circ}$ the motion of the fluid is completely defined by the complex potential

$$
W=-\frac{v R^{2}}{z-z_{c}}+\frac{q}{2 \pi} \ln \left(z-z_{q}\right)+\frac{q}{2 \pi} \ln \left(\frac{R^{2}}{z-z_{c}}-\overline{z_{q}-z_{c}}\right) .
$$

Here, $z=x+i y$ is the complex variable and $v=\dot{z}_{c}=v_{x}+i v_{y}$ is the velocity of the center of mass of the foil. 
We note that the first and second terms in (1.1) are the classical potentials of a circular cylinder moving with velocity $v$ and of a source of intensity $q$, respectively [34]. The third term is due to the Milne - Thomson theorem [47] and ensures the fulfillment of the impermeability condition on the boundary of the foil.

The force acting acting on the foil. To derive equations of motion of a circular foil, it is necessary to determine the force acting on it from the fluid. For the system treated here this force can be calculated using the formula proposed by Sedov in [55]

$$
F_{x}+i F_{y}=\overline{\frac{i \rho}{2} \oint_{L}\left(\frac{d W}{d z}\right)^{2} d z}+\frac{d}{d t}\left(\rho \frac{d S z_{c}}{d t}+i \rho \oint_{L} z \frac{d W}{d t} d z\right)
$$

where $\rho$ is the density of the fluid, $S=\pi R^{2}$ is the area of the foil, and $F_{x}$ and $F_{y}$ are the projections of the force onto the axes $O x$ and $O y$, respectively. By virtue of assumption $2^{\circ}$, the terms in (1.2) related to circulation are ignored.

To calculate the force acting on the foil, we assume that the positions of the source $z_{q}$ and of the geometric center of the foil $z_{c}$ depend on time $t$ and that the strength $q$ depends on time $t$, coordinates $z_{q}, z_{c}$ and velocities $\dot{z}_{q}, \dot{z}_{c}$. Then the projections $F_{x}$ and $F_{y}$ of the force onto the axes of the fixed coordinate system $O x y$ take the form

$$
\begin{aligned}
& F_{x}=\frac{\rho R^{2}}{2 \pi h^{2}\left|z_{s}\right|^{4}}\left(2 \pi q h^{2} \operatorname{Re}\left[\dot{\overline{z_{q}}} z_{s}^{2}\right]-2 h^{2}\left|z_{s}\right|^{2} \dot{q} \pi x_{s}-q^{2}\left|z_{s}\right|^{2} x_{s}\right)-\rho R^{2} \pi \dot{v}_{x} \\
& F_{y}=\frac{\rho R^{2}}{2 \pi h^{2}\left|z_{s}\right|^{4}}\left(2 \pi q h^{2} \operatorname{Im}\left[\dot{\overline{z_{q}}} z_{s}^{2}\right]-2 h^{2}\left|z_{s}\right|^{2} \dot{q} \pi y_{s}-q^{2}\left|z_{s}\right|^{2} y_{s}\right)-\rho R^{2} \pi \dot{v}_{y}
\end{aligned}
$$

where $z_{s}=z_{q}-z_{c}=x_{s}+i y_{s}, h^{2}=R^{2}-\left|z_{s}\right|^{2}$, and the dot denotes the total time derivative. The last terms in the expressions (1.3) are due to the effect of added masses.

In the general case where the strength $q$ depends on the coordinates $z_{q}, z_{c}$ and the velocities $\dot{z}_{q}, \dot{z}_{c}$, the force acting on the foil is not potential. In this case, the system may exhibit attracting regimes. This effect will be shown in Section 2.

Remark 1. The torque acting on the foil and calculated relative to its geometric center is zero. This is due to the fact that at each point of the contour the normal is directed to the center of the foil.

Remark 2. In the case of a fixed foil $\left(\dot{z}_{c}=0\right)$ and a fixed source with constant strength $\left(\dot{z}_{q}=0, \dot{q}=0\right.$ ) the force acting on the foil is always directed to the source and does not depend on the sign of $q$. This paradox of the model of an ideal fluid was first discovered in the classical work [47].

Equations of motion. In the case where the strength $q$ is a given function of time only, the force acting on the foil will be potential. In this case, the equations of motion of the center of mass of the foil can be represented in the canonical Hamiltonian form

$$
\dot{x}_{c}=\frac{\partial H}{\partial p_{x}}, \quad \dot{p}_{x}=-\frac{\partial H}{\partial x}, \quad \dot{y}_{c}=\frac{\partial H}{\partial p_{y}}, \quad \dot{p}_{y}=-\frac{\partial H}{\partial y},
$$

with the Hamiltonian explicitly depending on time

$$
H=\frac{A\left(p_{x}^{2}+p_{y}^{2}\right)}{2}-\frac{\rho R^{2} q}{\left|z_{s}\right|^{2}}\left(x_{s} \dot{x}_{q}+y_{s} \dot{y}_{q}\right)-\rho R^{2} \dot{q} \ln \left|z_{s}\right|+\frac{\rho q^{2}}{4 \pi} \ln \left(1-\frac{R^{2}}{\left|z_{s}\right|^{2}}\right) .
$$


Here, $m$ is the mass of the unit of length of the cylinder, $A^{-1}=m+\rho \pi R^{2}$ is the sum of the inertial and added masses of the foil, and $p_{x}=A^{-1} v_{x}$ and $p_{y}=A^{-1} v_{y}$ are the projections of the linear momentum of the foil + fluid system onto the axes $O x$ and $O y$, respectively. The equations of motion (1.4) can be explicitly written as

$$
\begin{aligned}
& \dot{x}_{c}=A p_{x}, \quad \dot{p}_{x}=\frac{\rho R^{2}}{2 \pi h^{2}\left|z_{s}\right|^{4}}\left(2 \pi q h^{2} \operatorname{Re}\left[\dot{\overline{z_{q}}} z_{s}^{2}\right]-2 h^{2}\left|z_{s}\right|^{2} \dot{q} \pi x_{s}-q^{2}\left|z_{s}\right|^{2} x_{s}\right), \\
& \dot{y}_{c}=A p_{y}, \quad \dot{p}_{y}=\frac{\rho R^{2}}{2 \pi h^{2}\left|z_{s}\right|^{4}}\left(2 \pi q h^{2} \operatorname{Im}\left[\dot{\overline{z_{q}}} z_{s}^{2}\right]-2 h^{2}\left|z_{s}\right|^{2} \dot{q} \pi y_{s}-q^{2}\left|z_{s}\right|^{2} y_{s}\right) .
\end{aligned}
$$

Below we consider the motion of a circular foil in the field of a fixed source with constant and variable strength.

\section{$\S 2$. Stability of the circular motion of the foil}

Fixed source of constant strength. Consider the motion of a cylinder in the field of a fixed source of constant strength $\left(\dot{z}_{q}=0, \dot{q}=0\right)$. Due to the arbitrariness of the choice of the origin of the coordinate system $O x y$ we can assume that the source is located at the point $z_{q}=0$. In this case, it is more convenient to describe the position of the foil relative to the source by the polar coordinates

$$
s=\left|z_{c}\right|=\sqrt{x_{c}^{2}+y_{c}^{2}}, \quad \vartheta=\arg z_{c}, \quad s \in(R,+\infty), \quad \vartheta \in[-\pi, \pi) .
$$

We define the generalized momenta corresponding to the coordinates $s, \vartheta$ as

$$
p_{s}=A^{-1} \dot{s}, \quad p_{\vartheta}=A^{-1} s^{2} \dot{\vartheta}
$$

The Poisson bracket of the new variables (2.1), (2.2) remains canonical and the Hamiltonian (1.5) takes the form

$$
H=\frac{A}{2}\left(p_{s}^{2}+\frac{p_{\vartheta}^{2}}{s^{2}}\right)+\frac{\rho q^{2}}{4 \pi} \ln \left(1-\frac{R^{2}}{s^{2}}\right)
$$

Since the Hamiltonian (2.3) does not depend on the variable $\vartheta$, the generalized (angular) momentum $p_{\vartheta}$ is preserved along the trajectories of the system $[1,50]$. Thus, the system under consideration admits the angular momentum integral

$$
F=p_{\vartheta}
$$

On the fixed level set $F=f$ of the integral (2.4) the equations of motion reduce to a system with one degree of freedom

$$
\dot{s}=\left.\frac{\partial H}{\partial p_{s}}\right|_{p_{\vartheta}=f}=A p_{s}, \quad \dot{p}_{s}=-\left.\frac{\partial H}{\partial s}\right|_{p_{\vartheta}=f}=\frac{A f^{2}}{s^{3}}-\frac{\rho R^{2} q^{2}}{2 \pi s\left(s^{2}-R^{2}\right)} .
$$

We note that, to reconstruct the motion of the foil on the plane $(x, y)$, we need to supplement equations (2.5) with the quadrature

$$
\vartheta(t)=\vartheta(0)+A f \int_{0}^{t} \frac{d \tau}{s^{2}(\tau)} .
$$


It is easy to show that, when $|f|>|q| R \sqrt{\frac{\rho}{2 \pi A}}$, equations (2.5) possess the saddle fixed point

$$
s=R|f| \sqrt{\frac{2 \pi A}{2 \pi A f^{2}-\rho q^{2} R^{2}}}, \quad p_{s}=0 .
$$

The trajectory of the geometric center of the foil on the plane $(x, y)$, which corresponds to the fixed point (2.6), is the circle

$$
x(t)=x_{q}+s_{0} \cos \left(\vartheta(0)+\frac{A f}{s_{0}^{2}} t\right), \quad y(t)=y_{q}+s_{0} \sin \left(\vartheta(0)+\frac{A f}{s_{0}^{2}} t\right) .
$$

We shall call this motion circular. Next, we address the question of its stabilization.

Fixed source of variable strength. It turns out that the unstable circular motion described above can be stabilized by controlling the intensity of the source via feedback.

As in the previous case, we will specify the position of the foil relative to the source by the polar coordinates (2.1), which correspond to the generalized momenta (2.2). In this case, the Hamiltonian (1.5) becomes

$$
H=\frac{A}{2}\left(p_{s}^{2}+\frac{p_{\vartheta}^{2}}{s^{2}}\right)+\frac{\rho q^{2}}{4 \pi} \ln \left(1-\frac{R^{2}}{s^{2}}\right)-\rho R^{2} \dot{q} \ln s .
$$

It is easy to see that, when $\dot{q} \neq 0$, the system also admits the angular momentum integral (2.4). The equations of motion on the fixed level set $F=f$ of the integral (2.4) take the form

$$
\dot{s}=A p_{s}, \quad \dot{p}_{s}=\frac{A f^{2}}{s^{3}}-\frac{\rho R^{2} q^{2}}{2 \pi s\left(s^{2}-R^{2}\right)}+\frac{\rho R^{2} \dot{q}}{s} .
$$

As control it is convenient to choose the derivative $\dot{q}$ since this quantity appears in equation (2.8) linearly. To prove the controllability and synthesis of feedback, it is convenient to make the following change of variables:

$$
s=s, \quad p_{s}=\frac{\mathcal{P}}{s} .
$$

Taking the change of variables (2.9) into account, equations (2.8) take the form

$$
\dot{s}=\frac{A \mathcal{P}}{s}, \quad \dot{\mathcal{P}}=\frac{A\left(\mathcal{P}^{2}+f^{2}\right)}{s^{2}}-\frac{\rho R^{2} q^{2}}{2 \pi\left(s^{2}-R^{2}\right)}+\rho R^{2} u, \quad \dot{q}=u,
$$

where $u$ is the control action. The phase space of the controlled system (2.10) is threedimensional. We denote it by $\mathcal{Q}=\{(s, \mathcal{P}, q) \mid s>R\}$.

In the absence of control $(u=0)$ the system (2.10) admits a one-parameter family of fixed points

$$
s=s_{0}=R|f| \sqrt{\frac{2 \pi A}{2 \pi A f^{2}-\rho q_{0}^{2} R^{2}}}, \quad \mathcal{P}=0, \quad q=q_{0},
$$

where $q_{0}$ is the parameter that satisfies the following inequality:

$$
0<\left|q_{0}\right|<\frac{|f|}{R} \sqrt{\frac{2 \pi A}{\rho}} .
$$


We carry out an analysis of the controllability and a synthesis of feedback by analogy with [44]. For this we fix the quantity $q_{0}$ and linearize equations (2.10) in a neighborhood of the corresponding fixed point of the family (2.11):

$$
\begin{gathered}
\dot{\boldsymbol{w}}=\mathbf{J} \boldsymbol{w}+\boldsymbol{b} u, \\
\boldsymbol{w}=\left(\begin{array}{c}
\delta s \\
\delta \mathcal{P} \\
\delta q
\end{array}\right), \quad \mathbf{J}=\left(\begin{array}{ccc}
0 & \alpha & 0 \\
\beta & 0 & \gamma \\
0 & 0 & 0
\end{array}\right), \quad \boldsymbol{b}=\left(\begin{array}{c}
0 \\
\rho R^{2} \\
1
\end{array}\right), \\
\alpha=\frac{A}{s_{0}}, \quad \beta=-\frac{2 A f^{2}}{s_{0}^{3}}+\frac{\rho R^{2} s_{0} q_{0}^{2}}{\pi\left(s_{0}^{2}-R^{2}\right)^{2}}, \quad \gamma=-\frac{\rho R^{2} q_{0}}{\pi\left(s_{0}^{2}-R^{2}\right)} .
\end{gathered}
$$

Here, the components of the vector $\boldsymbol{w}$ define the deviation from the fixed point $\left(s_{0}, 0, q_{0}\right)$.

The Jacobian $\mathbf{J}$ possesses the following set of eigenvalues and right-handed eigenvectors:

$$
\begin{gathered}
\lambda_{1}=0, \quad \lambda_{2}=\sqrt{\alpha \beta}, \quad \lambda_{3}=-\sqrt{\alpha \beta} \\
\boldsymbol{e}_{1}=\left(\begin{array}{c}
-\gamma \\
0 \\
\beta
\end{array}\right), \quad \boldsymbol{e}_{2}=\left(\begin{array}{c}
\alpha \\
\sqrt{\alpha \beta} \\
0
\end{array}\right), \quad \boldsymbol{e}_{3}=\left(\begin{array}{c}
\alpha \\
-\sqrt{\alpha \beta} \\
0
\end{array}\right) .
\end{gathered}
$$

We note that the eigenvalues $\lambda_{2}$ and $\lambda_{3}$ are real and the zero eigenvalue $\lambda_{1}$ corresponds to a shift in the parameter $q_{0}$ of the family of fixed points (2.11).

Since the system (2.12) is a linear system with constant coefficients, its controllability can be investigated on the basis of the Kalman criterion [25]. According to this criterion, the system (2.12) will be completely controllable if the following condition is satisfied:

$$
\operatorname{det}\left\|\boldsymbol{b}, \mathbf{J} \boldsymbol{b}, \mathbf{J}^{2} \boldsymbol{b}\right\|=\frac{2 \rho^{2} q_{0}^{2} A^{2} f^{2} R^{4}\left(2 m A f^{2}+\rho^{2} R^{4} q_{0}^{2}\right)}{s_{0}^{5}\left(s_{0}^{2}-R^{2}\right)^{2}\left(2 \pi A f^{2}-\rho R^{2} q_{0}^{2}\right)^{2}} \neq 0 .
$$

It can be seen from the expression (2.14) that the linearized system is completely controllable. Thus, it turns out to be possible to construct a control that leads the system (2.12) to a zero solution.

We will construct a control via feedback in the form of a linear combination of deviations $\delta s$, $\delta \mathcal{P}, \delta q$ :

$$
u=\boldsymbol{f}^{T} \cdot \boldsymbol{w}, \quad \boldsymbol{f}=\left(f_{1}, f_{2}, f_{3}\right)^{T}=\text { const } .
$$

Substituting the expression (2.15) into equation (2.12), we obtain

$$
\dot{\boldsymbol{w}}=\mathbf{J}_{f} \boldsymbol{w}, \quad \mathbf{J}_{f}=\mathbf{J}+\boldsymbol{b} \cdot \boldsymbol{f}^{T}
$$

It can be seen from equations (2.16) that, to stabilize the zero solution, it is necesary to choose the coefficients $f_{i}(i=1,2,3)$ in such a way that all eigenvalues of the matrix $\mathbf{J}_{f}$ have a negative real part. In this case, the zero solution to the system (2.12) becomes asymptotically stable and the solution (2.7) to the initial system (1.6) becomes a limit cycle.

In the general case, the eigenvalues of the matrix $\mathbf{J}_{f}$ are given by cumbersome expressions. Nevertheless, their analysis can be performed in some special cases. For this, it is convenient to pass to the basis of the eigenvectors of the matrix $\mathbf{J}$ by making the following change of variables:

$$
\boldsymbol{y}=\mathbf{K}^{-1} \boldsymbol{w}
$$


where $\mathbf{K}$ is the matrix whose columns are the eigenvectors (2.13). Then equations (2.16) take the form

$$
\begin{gathered}
\dot{\boldsymbol{y}}=\boldsymbol{\Omega} \boldsymbol{y}, \quad \boldsymbol{\Omega}=\boldsymbol{\Lambda}+\widetilde{\boldsymbol{b}} \cdot \boldsymbol{g}^{T} \\
\boldsymbol{\Lambda}=\operatorname{diag}\left(\lambda_{1}, \lambda_{2}, \lambda_{3}\right), \quad \widetilde{\boldsymbol{b}}=\mathbf{K}^{-1} \boldsymbol{b}, \quad \boldsymbol{g}^{T}=\boldsymbol{f}^{T} \mathbf{K} .
\end{gathered}
$$

Remark 3. It is easy to show that the following inequalities hold for the components of the vector $\widetilde{b}$ :

$$
\widetilde{b}_{1}>0, \quad \widetilde{b}_{2}\left(-q_{0}\right)=-\widetilde{b}_{3}\left(q_{0}\right), \quad \widetilde{b}_{2}\left(q_{0}\right) q_{0}<0, \quad \widetilde{b}_{3}\left(q_{0}\right) q_{0}<0, \quad \widetilde{b}_{2}>\widetilde{b}_{3} .
$$

Let us consider special cases where one of the coefficients $g_{i}(i=1,2,3)$ is zero.

1. When $g_{1}=0$, the matrix $\mathbf{J}_{f}$ possesses the following set of eigenvalues:

$$
\mu_{1}=0, \quad \mu_{2,3}=\frac{1}{2}\left(\widetilde{b}_{2} g_{2}+\widetilde{b}_{3} g_{3} \pm \sqrt{\left(\widetilde{b}_{2} g_{2}+\widetilde{b}_{3} g_{3}\right)^{2}+4 \lambda_{2}\left(\widetilde{b}_{2} g_{2}-\widetilde{b}_{3} g_{3}+\lambda_{2}\right)}\right) .
$$

Thus, when $g_{1}=0$, the eigenvalue $\mu_{1}$ of the matrix $\mathbf{J}_{f}$ is zero, i.e., feedback does not ensure the asymptotic stabilization of the zero solution to equations (2.16).

2. When $g_{2}=0$, the matrix $\mathbf{J}_{f}$ possesses the following set of eigenvalues:

$$
\mu_{1}=\lambda_{2}, \quad \mu_{2,3}=\frac{1}{2}\left(\widetilde{b}_{1} g_{1}+\widetilde{b}_{3} g_{3}-\lambda_{2} \pm \sqrt{\left(\widetilde{b}_{1} g_{1}+\widetilde{b}_{3} g_{3}\right)^{2}+\lambda_{2}\left(2 \widetilde{b}_{1} g_{1}-2 \widetilde{b}_{3} g_{3}+\lambda_{2}\right)}\right) .
$$

It is obvious that, when $g_{2}=0$, the eigenvalue $\mu_{1}$ of the matrix $\mathbf{J}_{f}$ is positive. Thus, feedback does not ensure the asymptotic stabilization of the zero solution to equations (2.16) either.

3. When $g_{3}=0$, the matrix $\mathbf{J}_{f}$ possesses the following set of eigenvalues:

$$
\mu_{1}=\lambda_{3}, \quad \mu_{2,3}=\frac{1}{2}\left(\widetilde{b}_{1} g_{1}+\widetilde{b}_{2} g_{2}+\lambda_{2} \pm \sqrt{\left(\widetilde{b}_{1} g_{1}+\widetilde{b}_{2} g_{2}+\lambda_{2}\right)^{2}-4 \widetilde{b}_{1} g_{1} \lambda_{2}}\right) .
$$

It follows from (2.18) that the eigenvalue $\mu_{1}$ is always negative. According to the expressions for $\mu_{2,3}$ and to inequalities (2.17), the real part of the eigenvalues $\mu_{2,3}$ will be negative if the coefficients $g_{1}, g_{2}$ satisfy the following inequality:

$$
\widetilde{b}_{1} g_{1}+\widetilde{b}_{2} g_{2}+\lambda_{2}<0 \text {. }
$$

Coming back to the initial basis and to the initial variables, we obtain the following feedback:

$$
u=\frac{g_{2}}{2 \alpha}\left(s-s_{0}\right)+\frac{g_{2}}{2 \sqrt{\alpha \beta}} s p_{s}+\left(\frac{g_{1}}{\beta}+\frac{g_{2} \gamma}{2 \alpha \beta}\right)\left(q-q_{0}\right) .
$$

The control (2.19) was constructed using linearized equations and, with $q_{0}$ given, guarantees the stabilization of the fixed point (2.6) only for small deviations from it. Nonetheless, numerical experiments show that the feedback (2.19) makes it possible to stabilize the circular motion of the foil even with large deviations.

We investigate numerically the behavior of the system with the feedback (2.19) depending on the initial conditions with the following parameter values:

$$
m=1, \quad R=1, \quad \rho=1, \quad q_{0}=1, \quad f=1, \quad g_{1}=0.8, \quad g_{2}=3 .
$$




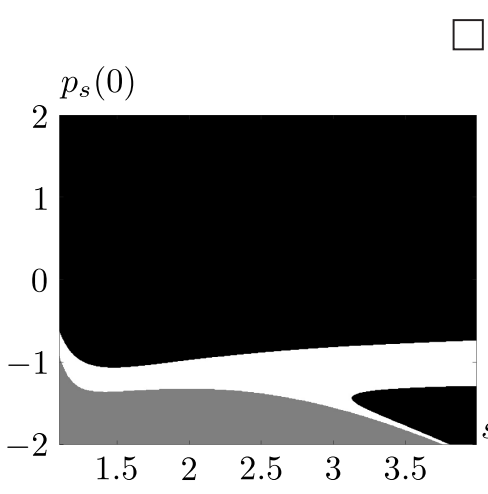

(a) $q(0)=-1$ fixed point

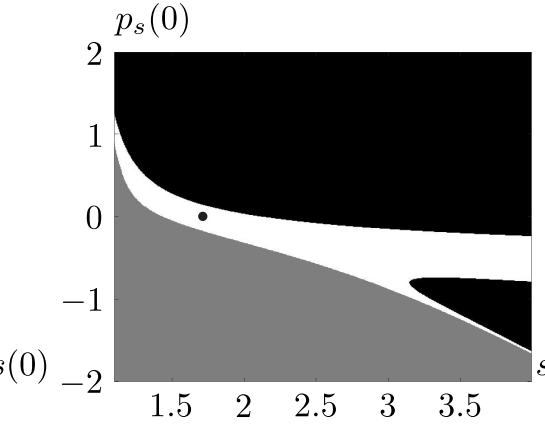

(b) $q(0)=1$ infinity
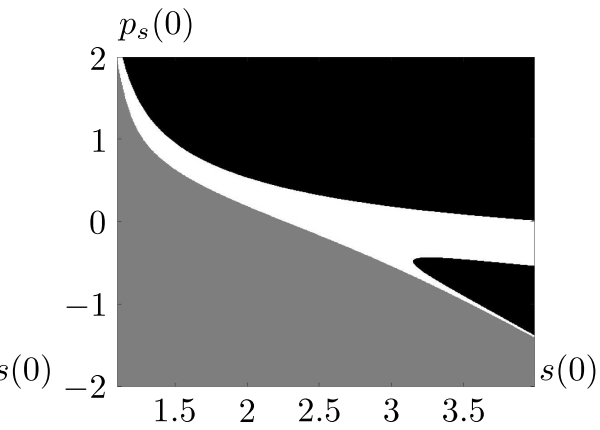

(c) $q(0)=2$

Figure 2: Sections of the domain of attraction for different values of $q(0)$ and the parameter values (2.20). The black marker in panel (b) denotes a fixed point.

Figure 2 shows the domains of attraction to the fixed point (white), domains of attraction to the source (gray) and the domains of realization of noncompact trajectories (black) for different initial intensity values of the source $q(0)$ on the plane of initial conditions $\left(s(0), p_{s}(0)\right)$.

For the parameter values (2.20) the eigenmatrices $\mathbf{J}_{f}$ have the following eigenvalues:

$$
\mu_{1} \approx-0.0837, \quad \mu_{2,3} \approx-0.5708 \pm 1.0108 i
$$

Since two eigenvalues have a nonzero imaginary part, the process of stabilization can be accompanied by oscillations of the values of the phase variables (see Fig. 3).

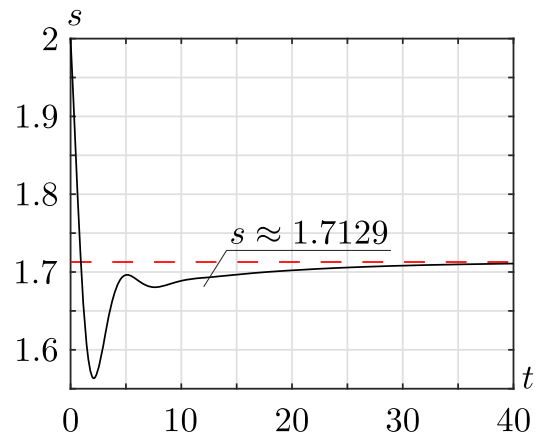

(a)

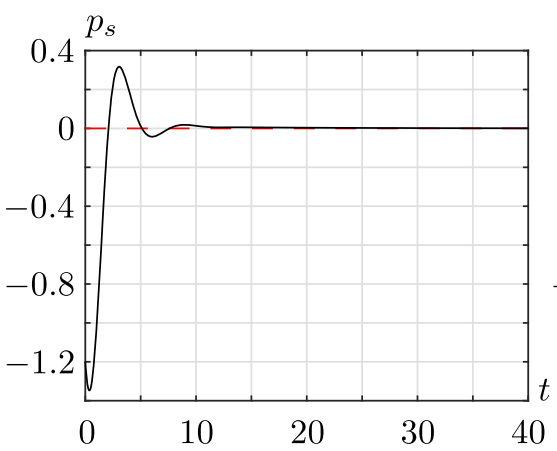

(b)

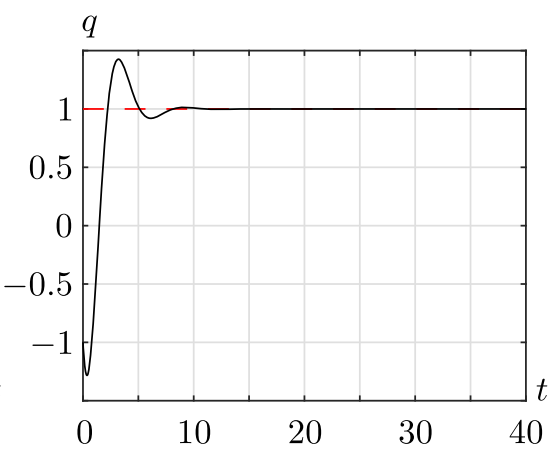

(c)

Figure 3: Change in the phase variables $s, p_{s}, q$ for the parameter values (2.20) and the initial conditions $s(0)=2, p_{s}(0)=-1.2, q(0)=-1$

We note that, by a suitable choice of the coefficients $g_{1}, g_{2}$, one can achieve an oscillation-free process of stabilization (see Fig. 4).

\section{§3. Conclusion}

To conclude, we point out a number of open problems:

1. Bifurcation analysis of dynamics in the case of a fixed source of constant strength.

2. Construction of feedback which ensures expansion of the domain of attraction to a fixed point.

3. Generalization of the model to the case of an arbitrary number of sources and vortex sources. 


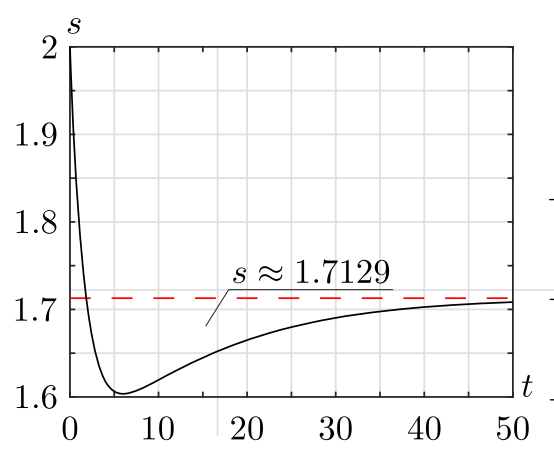

(a)

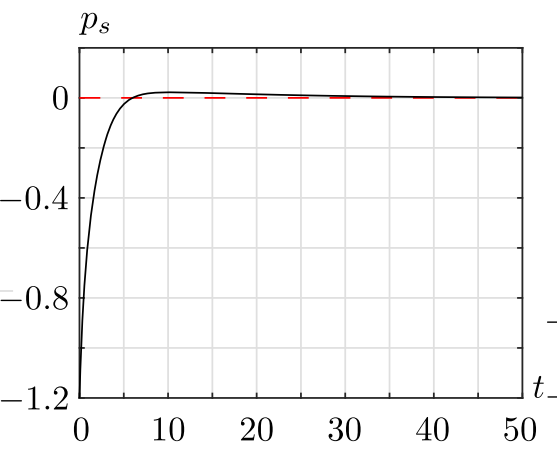

(b)

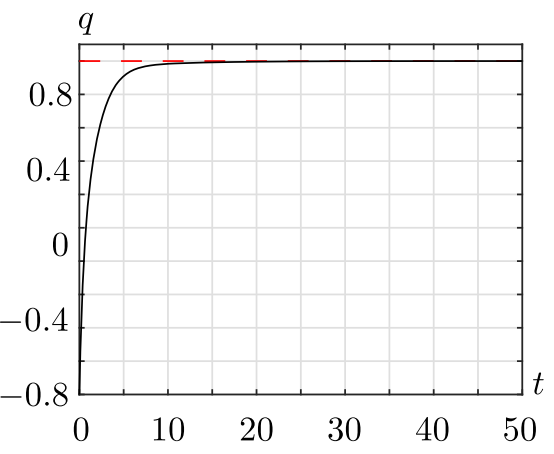

(c)

Figure 4: Change in the phase variables $s, p_{s}, q$ for the parameter values $m=1, R=1, \rho=1$, $q_{0}=1, f=1, g_{1}=0.7, g_{2}=3$ and the initial conditions $s(0)=2, p_{s}(0)=-1.2, q(0)=-1$. The matrix $\mathbf{J}_{f}$ possesses the following set of eigenvalues $\mu_{1} \approx-0.0837, \mu_{2} \approx-2.7208$, $\mu_{3} \approx-0.4333$

The authors extend their gratitude to I.A. Bizyaev, A.A. Kilin and I.S. Mamaev for valuable discussions and comments.

Funding. This work was supported by the Russian Foundation for Basic Research under grants 18-29-10050-mk and 18-08-00995-a.

\section{REFERENCES}

1. Arnold V.I. Mathematical methods of classical mechanics, New-York-Heidelberg-Berlin: Springer, 1974.

2. Belotelov V.N., Martynenko Yu.G. Control of spatial motion of an inverted pendulum mounted on a wheel pair, Mechanics of Solids, 2006, no. 6, pp. 10-28.

https://www.elibrary.ru/item.asp?id=9438845

3. Bizyaev I.A., Borisov A.V., Mamaev I.S. The dynamics of vortex sources in a deformation flow, Regular and Chaotic Dynamics, 2016, vol. 21, no. 3, pp. 367-376. https: //doi.org/10.1134/S1560354716030084

4. Bizyaev I.A., Borisov A.V., Mamaev I.S. The dynamics of three vortex sources, Regular and Chaotic Dynamics, 2014, vol. 19, no. 6, pp. 694-701.

https://doi.org/10.1134/S1560354714060070

5. Bogomolov V.A. The movement of an ideal fluid of constant density in the presence of sinks, Izv. $A N$ SSSR, Mekhanika Zhidkosti i Gaza, 1976, no. 4, pp. 21-27 (in Russian).

6. Borisov A.V., Kilin A.A., Mamaev I.S. Absolute and relative choreographies in the problem of the motion of point vortices in a plane, Doklady Mathematics, 2005, vol. 71, no. 1, pp. 139-144. https: / / www.elibrary.ru/item.asp?id=13491841

7. Borisov A.V., Mamaev I.S., Vetchanin E.V. Dynamics of a smooth profile in a medium with friction in the presence of parametric excitation, Regular and Chaotic Dynamics, 2018, vol. 23, no. 4, pp. 480-502. https: //doi.org/10.1134/S1560354718040081

8. Borisov A.V., Mamaev I.S., Vetchanin E.V. Self-propulsion of a smooth body in a viscous fluid under periodic oscillations of a rotor and circulation, Regular and Chaotic Dynamics, 2018, vol. 23, no. 7-8, pp. 850-874. https://doi.org/10.1134/S1560354718070043

9. Borisov A.V., Mamaev I.S., Bizyaev I.A. Three vortices in spaces of constant curvature: reduction, Poisson geometry, and stability, Regular and Chaotic Dynamics, 2018, vol. 23, no. 5, pp. 613-636. https://doi.org/10.1134/S1560354718050106

10. Borisov A.V., Kilin A.A. Stability of Thomson's configurations of vortices on a sphere, Regular and Chaotic Dynamics, 2000, vol. 5, no. 2, pp. 189-200.

https://doi.org/10.1070/RD2000v005n02ABEH000141 
11. Borisov A.V., Kilin A.A., Mamaev I.S. Transition to chaos in dynamics of four point vortices on a plane, Doklady Physics, 2006, vol. 51, no. 5, pp. 262-267.

https://doi.org/10.1134/S1028335806050089

12. Borisov A.V., Kilin A.A., Karavaev Y.L., Klekovkin A.V. Stabilization of the motion of a spherical robot using feedbacks, Applied Mathematical Modelling, 2019, vol. 69, pp. 583-592.

https://doi.org/10.1016/j.apm.2019.01.008

13. Borisov A.V., Mamaev I.S. On the problem of motion of vortex sources on a plane, Regular and Chaotic Dynamics, 2006, vol. 11, no. 4, pp. 455-466.

https://doi.org/10.1070/RD2006v011n04ABEH000363

14. Borisov A.V., Mamaev I.S., Ramodanov S.M. Motion of a circular cylinder and $n$ point vortices in a perfect fluid, Regular and Chaotic Dynamics, 2003, vol. 8, no. 4, pp. 449-462.

https://doi.org/10.1070/RD2003v008n04ABEH000257

15. Borisov A.V., Vetchanin E.V., Kilin A.A. Control of the motion of a triaxial ellipsoid in a fluid using rotors, Mathematical Notes, 2017, vol. 102, no. 4, pp. 455-464.

https://doi.org/10.1134/s0001434617090176

16. Chaplygin S.A. On the pressure of a plane-parallel flow on obstructing bodies (to the theory of an airplane), Mat. Sb., 1911, vol. 28, pp. 120-166 (in Russian).

http://mi.mathnet.ru/eng/msb6652

17. Childress S., Spagnolie S.E., Tokieda T. A bug on a raft: recoil locomotion in a viscous fluid, Journal of Fluid Mechanics, 2011, vol. 669, pp. 527-556.

https://doi.org/10.1017/S002211201000515X

18. Fridman A.A., Polubarinova P.Ya. On moving singularities of a flat motion of an incompressible fluid, Geofizicheskii Sbornik, 1928, vol. 5, issue 2, pp. 9-23 (in Russian).

19. Gebhard B., Ortega R. Stability of periodic solutions of the $N$-vortex problem in general domains, Regular and Chaotic Dynamics, 2019, vol. 24, no. 6, pp. 649-670.

https://doi.org/10.1134/S1560354719060054

20. Gonchar V.Yu., Ostapchuk P.N., Tur A.V., Yanovsky V.V. Dynamics and stochasticity in a reversible system describing interaction of point vortices with a potential wave, Physics Letters A, 1991, vol. 152, pp. 287-292. https://doi.org/10.1016/0375-9601(91)90107-J

21. Heughan D.M. An experimental study of a symmetrical aerofoil with a rear suction slot and a retractable flap, The Aeronautical Journal, 1953, vol. 57, no. 514, pp. 627-645.

https://doi.org/10.1017/S0368393100126719

22. Ivanova T.B., Kilin A.A., Pivovarova E.N. Controlled motion of a spherical robot with feedback. I, Journal of Dynamical and Control Systems, 2018, vol. 24, no. 3, pp. 497-510.

https://doi.org/10.1007/s10883-017-9387-2

23. Ivanova T.B., Kilin A.A., Pivovarova E.N. Controlled motion of a spherical robot with feedback. II, Journal of Dynamical and Control Systems, 2019, vol. 25, no. 1, pp. 1-16.

https://doi.org/10.1007/s10883-017-9390-7

24. Ivlev V.I., Misyurin S.Y., Nosova N.Y. Servopneumatic actuator of a robot with compensation for the mutual influence of movements of the degrees of mobility, Vestnik Udmurtskogo Universiteta. Matematika. Mekhanika. Komp'yuternye Nauki, 2018, vol. 28, issue 2, pp. 231-239.

https://doi.org/10.20537/vm180209

25. Kalman R.E., Falb P.L., Arbib M.A. Topics in mathematical system theory, New York: McGraw-Hill, 1969.

26. Kanso E., Marsden J.E., Rowley C.W., Melli-Huber J. Locomotion of articulated bodies in a perfect fluid, Journal of Nonlinear Science, 2005, vol. 15, pp. 255-289.

https://doi.org/10.1007/s00332-004-0650-9

27. Kasper W. Aircraft wing with vortex generation, U.S. Patent No. 3831885 (27 Aug 1974). https://patents.google.com/patent/US3831885A/en

28. Kelly S.D., Xiong H. Self-propulsion of a free hydrofoil with localized discrete vortex shedding: analytical modeling and simulation, Theoretical and Computational Fluid Dynamics, 2010, vol. 24, pp. 45-50. https://doi.org/10.1007/s00162-009-0174-x 
29. Kelly S.D., Hukkeri R.B. Mechanics, dynamics, and control of a single-input aquatic vehicle with variable coefficient of lift, IEEE Transactions on Robotics, 2006, vol. 22, no. 6, pp. 1254-1264. https://doi.org/10.1109/TRO.2006.882934

30. Kilin A.A., Vetchanin E.V. The contol of the motion through an ideal fluid of a rigid body by means of two moving masses, Russian Journal of Nonlinear Dynamics, 2015, vol. 11, no. 4, pp. 633-645. https://doi.org/10.20537/nd1504001

31. Kiselev L.V., Medvedev A.V. Comparative analysis and optimization of the dynamic properties of autonomous underwater robots of various designs and configurations, Podvodnye Issledovaniya $i$ Robototekhnika, 2012, no. 1 (13), pp. 24-35 (in Russian).

http://jmtp.febras.ru/journal/1-13-2012/24-35.pdf

32. Kiselev O.M. Stable feedback control of a fast wheeled robot, Russian Journal of Nonlinear Dynamics, 2018, vol. 14, no. 3, pp. 409-417. https: //doi.org/10.20537/nd180310

33. Klenov A.I., Kilin A.A. Influence of vortex structures on the controlled motion of an above-water screwless robot, Regular and Chaotic Dynamics, 2016, vol. 21, no. 7-8, pp. 927-938. https://doi.org/10.1134/S1560354716070145

34. Kochin N.E., Kibel I.A., Roze N.V. Theoretical hydrodynamics, New York: Wiley, 1964.

35. Koiller J., Castilho C., Rodrigues A.R. Vortex pairs on the triaxial ellipsoid: axis equilibria stability, Regular and Chaotic Dynamics, 2019, vol. 24, no. 1, pp. 61-79. https://doi.org/10.1134/S1560354719010039

36. Kozlov V.V., Ramodanov S.M. The motion of a variable body in an ideal fluid, Journal of Applied Mathematics and Mechanics, 2001, vol. 65, no. 4, pp. 579-587.

https://doi.org/10.1016/s0021-8928(01)00063-6

37. Kozlov V.V., Onishchenko D.A. The motion in a perfect fluid of a body containing a moving point mass, Journal of Applied Mathematics and Mechanics, 2003, vol. 67, no. 4, pp. 553-564. https://doi.org/10.1016/S0021-8928(03)90058-X

38. Kutta W.M. Auftriebskräfte in strömenden Flüssigkeiten, Illustr. Aeronaut. Mitteilungen, 1902, vol. 6, pp. 133-135.

39. Kurakin L.G., Ostrovskaya I.V. On the stability of Thomson's vortex $N$-gon and a vortex tripole/quadrupole in geostrophic models of Bessel vortices and in a two-layer rotating fluid: a review, Russian Journal of Nonlinear Dynamics, 2019, vol. 15, no. 4, pp. 533-542. https://doi.org/10.20537/nd190412

40. Lighthill M.J. On the squirming motion of nearly spherical deformable bodies through liquids at very small Reynolds numbers, Communications on Pure and Applied Mathematics, 1952, vol. 5, no. 2, pp. 109-118. https://doi.org/10.1002/cpa.3160050201

41. Lin X., Guo S. Development of a spherical underwater robot equipped with multiple vectored waterjet-based thrusters, Journal of Intelligent \& Robotic Systems, 2012, vol. 67, no. 3-4, pp. 307-321. https://doi.org/10.1007/s10846-012-9651-3

42. Mamaev I.S., Vetchanin E.V. The self-propulsion of a foil with a sharp edge in a viscous fluid under the action of a periodically oscillating rotor, Regular and Chaotic Dynamics, 2018, vol. 23, no. 7-8, pp. 875-886. https://doi.org/10.1134/S1560354718070055

43. Mason R.J. Fluid locomotion and trajectory planning for shape-changing robots, PhD Dissertation, Pasadena, California: California Institute of Technology, 2003, 264 p. https://doi.org/10.7907/MFM1-0866

44. Martynenko Yu.G., Formalskiy A.M. The theory of the control of a monocycle, Journal of Applied Mathematics and Mechanics, 2005, vol. 69, no. 4, pp. 516-528. https://doi.org/10.1016/j.jappmathmech.2005.07.003

45. Mazumdar A., Asada H.H. Pulse width modulation of water jet propulsion systems using high-speed Coanda-effect valves, Journal of Dynamic Systems, Measurement, and Control, 2013, vol. 135, no. 5, 051019, 11 pp. https://doi.org/10.1115/1.4024365

46. Michelin S., Llewellyn Smith S.G. An unsteady point vortex method for coupled fluid-solid problems, Theoretical and Computational Fluid Dynamics, 2009, vol. 23, no. 2, pp. 127-153.

https://doi.org/10.1007/s00162-009-0096-7 
47. Milne-Thomson L.M. Theoretical hydrodynamics, 4th ed., Macmillan \& Co. Ltd, 1962.

48. Nelson R., Protas B., Sakajo T. Linear feedback stabilization of point-vortex equilibria near a Kasper wing, Journal of Fluid Mechanics, 2017, vol. 827, pp. 121-154. https://doi.org/10.1017/jfm.2017.484

49. Novikov A.E., Novikov E.A. Vortex-sink dynamics, Physical Review E, 1996, vol. 54, no. 4, pp. 36813686. https://doi.org/10.1103/PhysRevE.54.3681

50. Olver P.J. Application of Lie groups to differential equations, 2nd ed., Grad. Texts in Math., vol. 107, Springer, 2000.

51. Ramodanov S.M. Motion of a circular cylinder and $N$ point vortices in a perfect fluid, Regular and Chaotic Dynamics, 2002, vol. 7, no. 3, pp. 291-298. https://doi.org/10.1070/RD2002v007n03ABEH000211

52. Ramodanov S.M., Tenenev V.A. Motion of a body with variable distribution of mass in a boundless viscous liquid, Russian Journal of Nonlinear Dynamics, 2011, vol. 7, no. 3, pp. 635-647 (in Russian). https://doi.org/10.20537/nd1103016

53. Ramodanov S.M., Tenenev V.A., Treschev D.V. Self-propulsion of a body with rigid surface and variable coefficient of lift in a perfect fluid, Regular and Chaotic Dynamics, 2012, vol. 17, no. 6, pp. 547-558. https://doi.org/10.1134/S1560354712060068

54. Rust I.C., Asada H.H. The eyeball ROV: design and control of a spherical underwater vehicle steered by an internal eccentric mass, 2011 IEEE International Conference on Robotics and Automation, IEEE, 2011, pp. 5855-5862. https://doi.org/10.1109/ICRA.2011.5979835

55. Sedov L.I. Ploskie zadachi gidrodinamiki $i$ aerodinamiki (Two-dimensional problems in hydro- and aeromechanics), Second Edition, Moscow: Gostekhizdat, 1950.

56. Shashikanth B.N., Marsden J.E., Burdick J.W., Kelly S.D. The Hamiltonian structure of a twodimensional rigid circular cylinder interacting dynamically with $N$ point vortices, Physics of Fluids, 2002, vol. 14, no. 3, pp. 1214-1227. https://doi.org/10.1063/1.1445183

57. Smith C.B. A solution for the lift and drag of airfoils with air inlets and suction slots, Journal of the Aeronautical Sciences, 1949, vol. 16, no. 10, pp. 581-589.

https://doi.org/10.2514/8.11863

58. Llewellyn Smith S.G. How do singularities move in potential flow? Physica D: Nonlinear Phenomena, 2011, vol. 240, no. 20, pp. 1644-1651.

https://doi.org/10.1016/j.physd.2011.06.010

59. Vetchanin E.V., Kilin A.A. Control of body motion in an ideal fluid using the internal mass and the rotor in the presence of circulation around the body, Journal of Dynamical and Control Systems, 2017, vol. 23, pp. 435-458. https : //doi.org/10.1007/s10883-016-9345-4

60. Vetchanin E.V., Kilin A.A. Controlled motion of a rigid body with internal mechanisms in an ideal incompressible fluid, Proceedings of the Steklov Institute of Mathematics, 2016, vol. 295, pp. 302-332. https://doi.org/10.1134/S0081543816080186

61. Vetchanin E.V., Kilin A.A. Control of the motion of an unbalanced heavy ellipsoid in an ideal fluid using rotors, Russian Journal of Nonlinear Dynamics, 2016, vol. 12, no. 4, pp. 663-674 (in Russian). https://doi.org/10.20537/nd1604009

62. Vetchanin E.V., Kazakov A.O. Bifurcations and chaos in the dynamics of two point vortices in an acoustic wave, International Journal of Bifurcation and Chaos, 2016, vol. 26, no. 4, 1650063. https://doi.org/10.1142/S0218127416500632

63. Vetchanin E.V., Mamaev I.S. Dynamics of two point vortices in an external compressible shear flow, Regular and Chaotic Dynamics, 2017, vol. 22, no. 8, pp. 893-908.

https://doi.org/10.1134/S1560354717080019 
Artemova Elizaveta Markovna, Ural Mathematical Center, Udmurt State University, ul. Universitetskaya, 1, Izhevsk, 426034, Russia.

ORCID: https: //orcid.org/0000-0001-7637-2757

E-mail: liz-artemova2014@yandex.ru

Vetchanin Evgeny Vladimirovich, Ural Mathematical Center, Udmurt State University, ul. Universitetskaya, 1, Izhevsk, 426034, Russia.

ORCID: https: //orcid.org/0000-0002-2617-7512

E-mail: eugene186@mail.ru

Citation: E. M. Artemova, E. V. Vetchanin. Control of the motion of a circular cylinder in an ideal fluid using a source, Vestnik Udmurtskogo Universiteta. Matematika. Mekhanika. Komp'yuternye Nauki, 2020, vol. 30, issue 4, pp. 604-617. 


\section{Е. М. Артемова, Е. В. Ветчанин}

Управление движением кругового цилиндра в идеальной жидкости с помощью источника

Ключевые слова: управление, идеальная жидкость, обратная связь, движение в присутствии источника.

УДК 532.5.011

DOI: $10.35634 / \mathrm{vm} 200405$

Рассмотрено движение кругового цилиндра в идеальной жидкости в поле неподвижного источника. Показано, что при постоянной интенсивности источника система обладает интегралом момента и интегралом энергии. Указаны условия, при которых уравнения движения, редуцированные на уровень интеграла момента, допускают неустойчивую неподвижную точку. Данная неподвижная точка соответствует круговому движению цилиндра вокруг источника. Построена обратная связь, обеспечивающая стабилизацию указанной неподвижной точки за счет изменения интенсивности источника.

Финансирование. Исследование выполнено при поддержке РФФИ в рамках грантов 18-29-10050-мк и 18-08-00995-а.

Поступила в редакцию 29.09 .2020

Артемова Елизавета Марковна, Уральский математический центр, Удмуртский государственный университет, 426034, Россия, г. Ижевск, ул. Университетская, 1.

ORCID: https: //orcid.org/0000-0001-7637-2757

E-mail: liz-artemova2014@yandex.ru

Ветчанин Евгений Владимирович, Уральский математический центр, Удмуртский государственный университет, 426034, Россия, г. Ижевск, ул. Университетская, 1.

ORCID: https: //orcid.org/0000-0002-2617-7512

E-mail: eugene186@mail.ru

Цитирование: Е. М. Артемова, Е. В. Ветчанин. Управление движением кругового цилиндра в идеальной жидкости с помощью источника // Вестник Удмуртского университета. Математика. Механика. Компьютерные науки. 2020. Т. 30. Вып. 4. С. 604-617. 